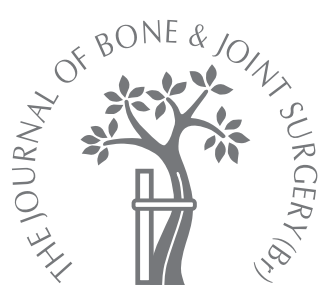

M. Olsen,

E. T. Davis,

J. P. Waddell,

E. H. Schemitsch

From the University

of Toronto, Toronto, Canada

\title{
Imageless computer navigation for placement of the femoral component in resurfacing arthroplasty of the hip
}

\begin{abstract}
We have investigated the accuracy of placement of the femoral component using imageless navigation in 100 consecutive Birmingham Hip Resurfacings. Pre-operative templating determined the native neck-shaft angle and planned stem-shaft angle of the implant. The latter were verified post-operatively using digital anteroposterior unilateral radiographs of the hip.

The mean neck-shaft angle determined before operation was $132.7^{\circ}\left(118^{\circ}\right.$ to $\left.160^{\circ}\right)$. The mean planned stem-shaft angle was a relative valgus alignment of $9.7^{\circ}$ (SD 2.6). The stemshaft angle after operation differed from that planned by a mean of $2.8^{\circ}$ (SD 2.0) and in $86 \%$ of cases the final angle measured within $\pm 5^{\circ}$ of that planned. We had no instances of notching of the neck or varus alignment of the implant in our series. A learning curve was observed in the time taken for navigation, but not for accurate placement of the implant.

Navigation in hip resurfacing may afford the surgeon a reliable and accurate method of placement of the femoral component.
\end{abstract}

Hip resurfacing presents an alternative to total hip replacement (THR) in the young adult. It conserves bone and has acceptable short- to medium-term results. ${ }^{1-7}$ Many of the problems associated with failure of resurfacing in previous generations have been resolved after improvements in manufacturing and surgical technique. However, concern about fracture of the femoral neck remains. Failure of resurfacing has been attributed to patient characteristics such as gender, bone density, height and weight as well as to mechanical factors including notching of the femoral neck, varus alignment of the implant and exposure of reamed cancellous bone. ${ }^{1,8-17}$ Several authors have suggested guidelines for implanting the femoral component by accurate preparation of the femoral head in order to minimise the likelihood of catastrophic failure. . $^{1,8,10,14}$

A successful outcome in hip resurfacing is largely dependent on the accuracy of the placement of the femoral component. ${ }^{14}$ The use of conventional guidewire alignment instruments can result in inconsistency between the planned position of the implant and the end result. ${ }^{18}$ Methods such as computer navigation have the potential to improve the accuracy of placement of the implant. ${ }^{19-28}$ We describe the methods and results of intra-operative imageless computer navigation for placement of the femoral component in the first 100 such procedures performed at our institution (St. Michael's Hospital, Toronto, Canada).

\section{Patients and Methods}

Between October 2005 and November 2007, 100 consecutive navigated hip resurfacings were performed in 94 patients by the senior surgeon (EHS). There were 77 men and 17 women. The primary diagnosis was osteoarthritis in 96 hips and avascular necrosis in four. The mean age of the patients at the time of surgery was 51.3 years (25 to 82$)$ and the mean body mass index was $29.5 \mathrm{~kg} / \mathrm{m}^{2}$ (20.4 to 51.9 ).

Before each procedure, pre-operative templating was carried out to determine the size of the implant and the anatomical axes. Modified digital anteroposterior (AP) unilateral radiographs of the hip were used showing the lateral pelvis and proximal two-thirds of the femur. In order to determine the axis of the femoral shaft, a line was drawn from the most distal visible diaphyseal mid-point through a point approximating to the piriformis fossa. This method is similar to those described in the literature and agrees with the navigation algorithm for determination of the diaphyseal axis. ${ }^{29-31}$ The neck axis was defined as a line drawn through the isthmus of the neck towards the lateral cortex intersecting the axis of the femoral shaft. The angle subtended by the axes of the femoral neck and shaft was the 


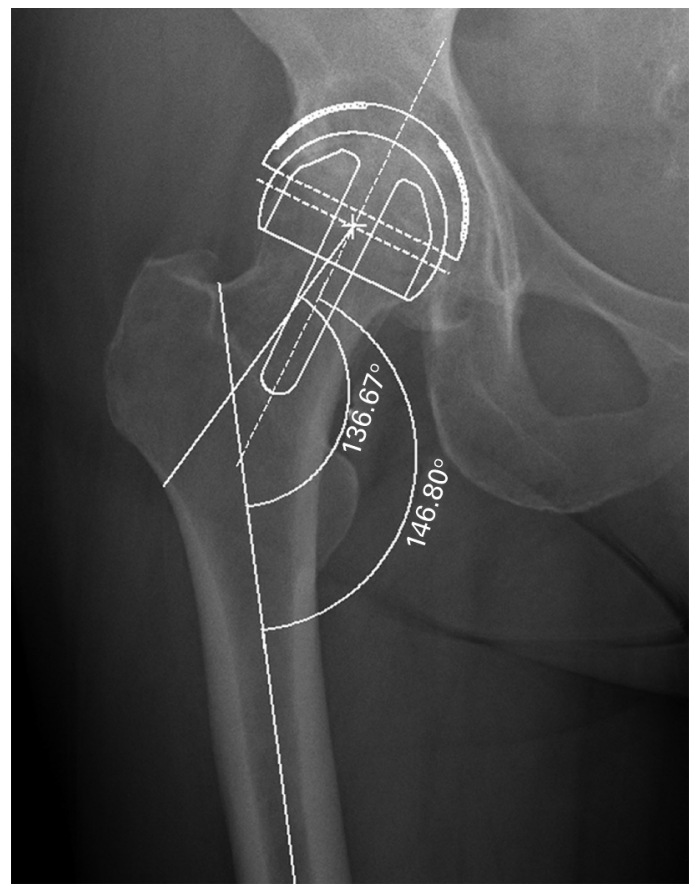

Fig. 1

Radiograph showing the typical pre-operative plan for hip resurfacing. The axes of the diaphysis and neck are drawn and a digital template of the femoral component is positioned in a relatively valgus position. The neck-shaft and stem-shaft angles are measured for use during intra-operative planning.

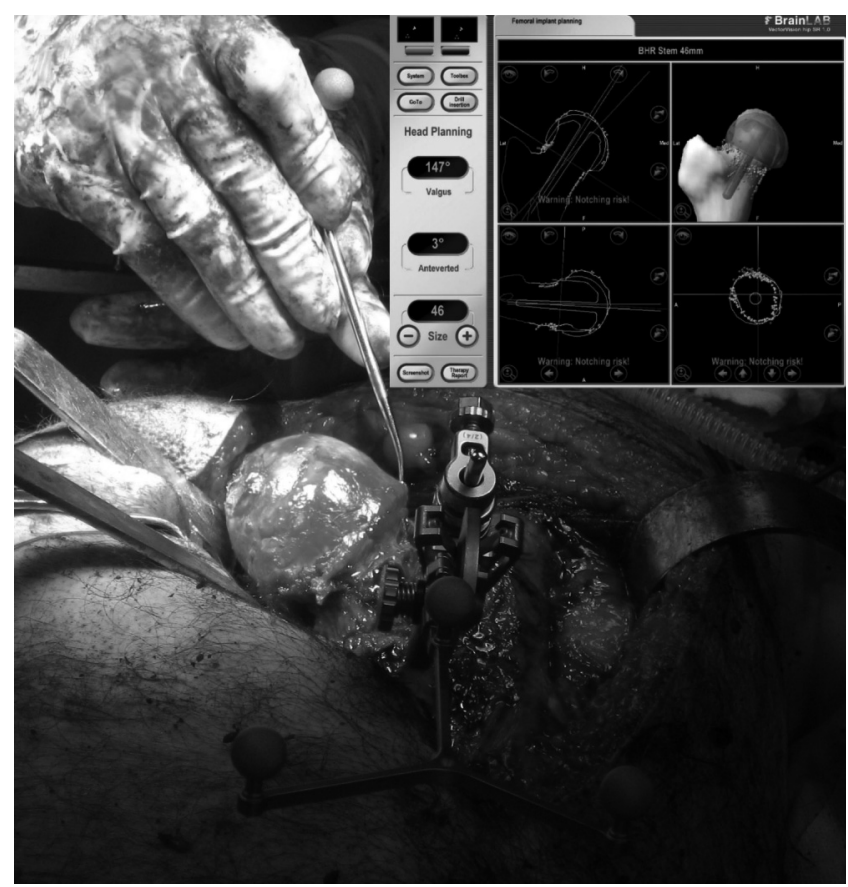

Fig. 2

Intra-operative photograph showing registration of the femoral head. The pointer is guided in concentric circles over the entire articular surface. The inset shows the navigation planning screen. neck-shaft angle. The sizes of the acetabular and femoral component were then selected using a templating software package (EndoMap version 2.01; Hectec GmbH, Niedervienbach, Germany). The femoral component was positioned in an appropriate amount of valgus orientation to the measured neck-shaft angle (Fig. 1). We routinely plan to position the stem to align with the medial calcar. The angle made by the stem and the axis of the femoral shaft was the planned stem-shaft angle. The values of these were recorded for use during intra-operative navigation. The resulting stem-shaft angle is generally approximated to $5^{\circ}$ to $10^{\circ}$ of relative valgus. This angulation has been recommended in the literature and has been shown to strengthen the resurfacing construct. ${ }^{8,10,12,14,32-34}$

Operative technique. A standard posterolateral approach was used in all cases with the patients in the lateral decubitus position. A full capsulotomy was completed upon dislocation of the femur. The acetabulum was reamed in the standard fashion, with the femur retracted anterosuperiorly, and the acetabular component was impacted in place. The femur was then internally rotated maximally and adducted, elevating the femoral head out of the wound. The head was prepared according to standard surgical protocol with the use of computer navigation for placement of the initial guidewire. In all cases we used the Birmingham Hip Resurfacing (Smith \& Nephew, Memphis, Tennessee), which is a hybrid resurfacing system using a press-fit acetabular component and a cemented femoral component. Imageless computer navigation. For intra-operative surgical planning and insertion of the initial guidewire, the Vector Vision imageless navigation system version 1.0 was used (BrainLAB, Heimstetten, Germany). The system acquires anatomical landmarks and surface data to generate a model upon which intra-operative planning can be conducted. It uses two cameras which transmit and receive reflected infrared light to triangulate the position of two optical arrays, a static array and a dynamic pointer. Each array is fixed with three reflective spheres. A $5 \mathrm{~mm} \mathrm{Schanz}$ pin is first drilled into the lesser trochanter and used to anchor the static array. Next, points at the medial and lateral femoral condyles and the piriformis fossa were acquired and used to delineate the diaphyseal axis. The superior head-neck junction is acquired along with clouds of points on the femoral head and the anterior, superior, posterior and inferior quadrants of the femoral neck (Fig. 2). Lastly, the anterosuperior part of the neck, the zone for superior notching is registered and provides enhanced visualisation of this critical area. The system calculates the neck-shaft angle, or the caput-collum-diaphysis angle, by fitting a plane to the superior and inferior clouds of points and calculating a mid-plane between the two. The inclination of the mid-plane to the diaphyseal axis is the computed 


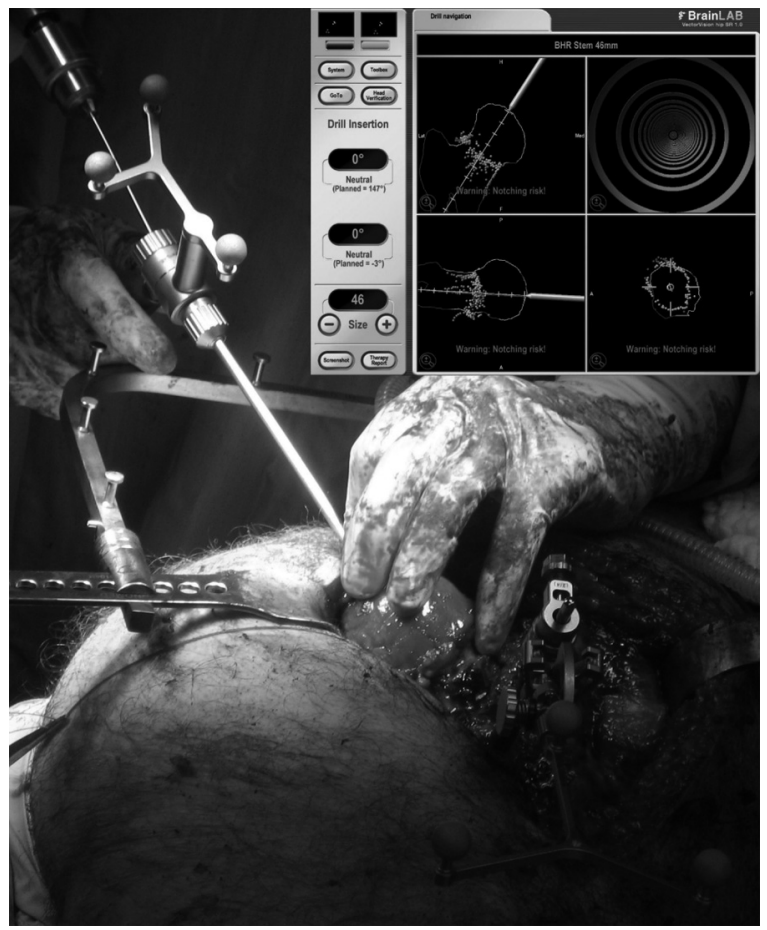

Fig. 3

Photograph showing insertion of the guidewire. The surgeon uses a navigated drill guide to drill the guidewire with real-time positional feedback (inset).

neck-shaft angle. The system also uses the registered data to determine the initial computed size and location of the implant. A computer-generated, patient-specific model is then created and verified for accuracy.

An intra-operative planning screen is used to adjust the size, location and angulation of the implant before insertion of the guidewire. A navigated drill guide is used to drill a $2.4 \mathrm{~mm}$ guidewire into the femoral head (Fig. 3). The drill guide provides real-time feedback of the position of the guidewire during drilling. The final location of the guidewire is verified using the same drill guide. The navigation time for the procedure is defined as the time between drilling the Schanz pin into the lesser trochanter and verification of the final position of the guidewire. After verification, a stylus is inserted over the guidewire to check for notching and sufficient resection of the head, and the remainder of the standard protocol is then followed for preparation of the femoral head.

Post-operative assessment. The stem-shaft angle of the femoral component was assessed on digital AP unilateral radiographs of the hip taken three months after operation. These were centred over the proximal femur and the lateral pelvis, showing the proximal two-thirds of the femur. A standard protocol was observed for positioning with the patient supine and the hip extended and in neutral rotation. Three patients were lost to follow-up by three months and their films taken before discharge were used for post-

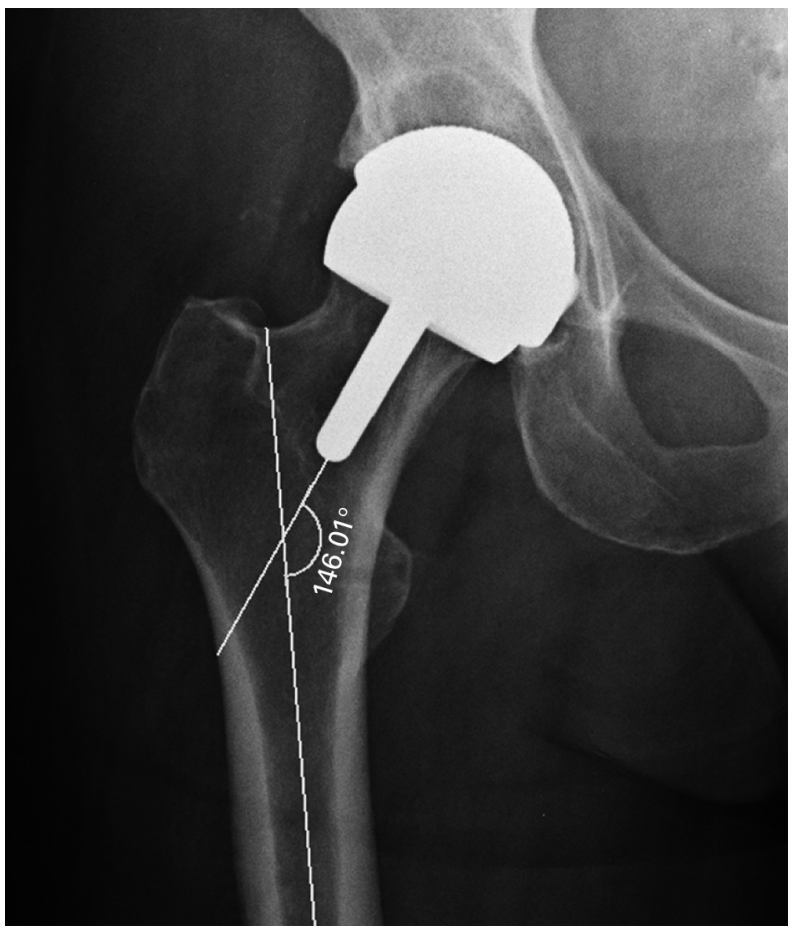

Fig. 4

Radiograph showing post-operative assessment of the stem-shaft angle of the femoral component.

operative assessment. The same observer (MO) undertook measurement of the stem-shaft angles of the femoral components. The diaphyseal axis was drawn in accordance with the pre-operative plan. A second line was drawn along the stem of the implant towards the lateral cortex and the larger angle subtended by the two intersecting lines was defined as the stem-shaft angle (Fig. 4). After operation this angle was considered to be acceptable if it did not exceed $5^{\circ}$ of varus relative to that planned. Angles in excess of $5^{\circ}$ of this were considered to be acceptable provided that the implant appeared to be well positioned with no evidence of notching of the neck.

Statistical analysis. The series was broken down into 20 discrete case intervals for analysis. The statistical software package SPSS (SPSS Inc., Chicago, Illinois) was used to perform one-way analysis of variance with Tukey post hoc analysis comparing differences in navigation time and accuracy of coronal alignment between each interval. Microsoft Excel (Microsoft Corporation, Redmond, Washington) was used to determine descriptive statistics for the series. A p-value of $<0.05$ was considered statistically significant.

\section{Results}

Pre-operative templating determined a mean neck-shaft angle of $132.7^{\circ}\left(118^{\circ}\right.$ to $\left.160^{\circ}\right)$. The mean planned stemshaft angle was $9.7^{\circ}$ of relative valgus to the original neck-shaft angle $\left(\mathrm{SD} 2.6^{\circ}\right)$. This value ranged from $0^{\circ}$ to 


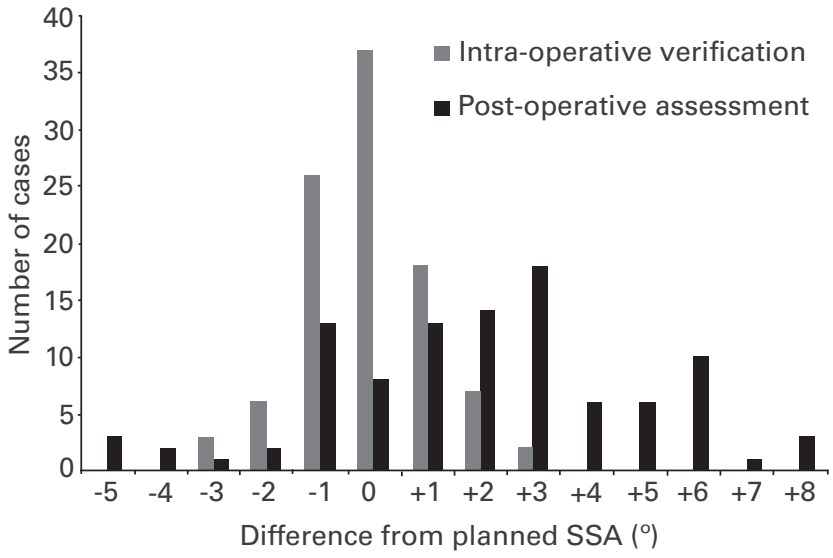

Fig. 5

Histogram showing the differences between the pre-operatively planned stem-shaft angle and the intra-operatively verified inclination of the guidewire and post-operatively assessed stem-shaft angle (SSA). Positive values indicate relative valgus and negative values relative varus.

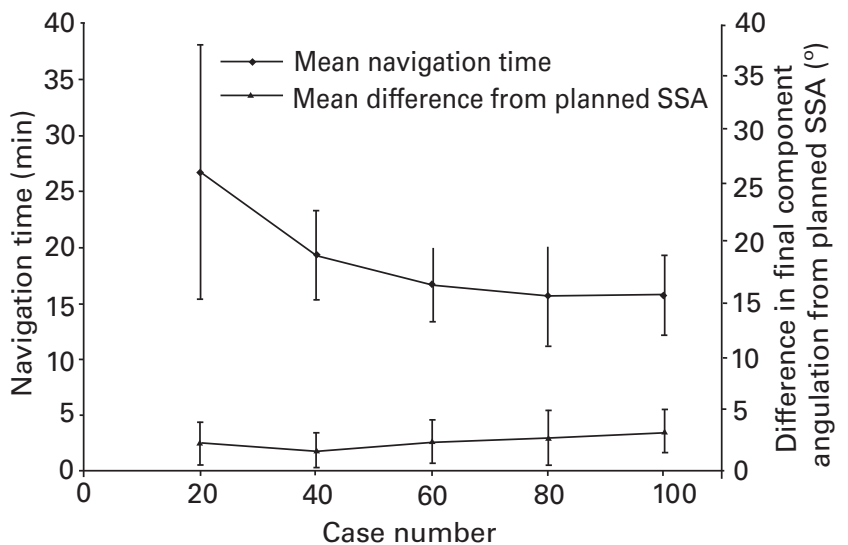

Fig. 6

Graph showing the navigation curve. The top curve illustrates the reduction in the mean navigation time in our series. There was a significant decrease in time between the first 20 cases and the remainder of the series. The lower curve shows the accuracy of the final stem-shaft angle of the femoral component (SSA, stem-shaft angle). $16^{\circ}$ of relative valgus based on the anatomy of the patient and the measured neck-shaft angle. Intra-operative verification of the position of the guidewire after insertion into the femoral head yielded a mean difference from the pre-operatively planned stem-shaft angle of $0.9^{\circ}\left(0^{\circ}\right.$ to $\left.3^{\circ}\right)$. This measure represented the error introduced by the surgeon during the insertion of the guidewire. Comparison between the inclination of the guidewire verified intra-operatively and that measured after the procedure showed a mean difference of $3.0^{\circ}\left(0^{\circ}\right.$ to $\left.9^{\circ}\right)$. Because of the low degree of error in drilling the guidewire, this difference was similar to the overall difference between the planned stem-shaft angle and that measured on the post-operative radiographs which yielded a mean difference of $2.8^{\circ}\left(0^{\circ}\right.$ to $\left.8^{\circ}\right)$. The final stem-shaft angle was considered to be acceptable in all cases and measured within $\pm 3^{\circ}$ of that planned in $69 \%$ cases $(69 \%)$ and $\pm 5^{\circ}$ of the intended position in 86 cases $(86 \%)$ (Fig. 5). Of the remaining 14 cases, all measurements erred in valgus, of which none exceeded $8^{\circ}$. When compared with the pre-operative neck-shaft angle, the final stem-shaft angle showed a mean of $11.7^{\circ}$ of relative valgus $\left(1^{\circ}\right.$ to $22^{\circ}, 95 \%$ confidence interval (CI) 10.9 to 12.5 ).

The most common size of femoral component implanted in women was $46 \mathrm{~mm}$ (44 to 50) and in men $54 \mathrm{~mm}$ (46 to 60 ). The sizes of the corresponding acetabular components were $52 \mathrm{~mm}$ (50 to 56) for women and $60 \mathrm{~mm}$ for men (54 to 64). No sign of notching of the superior cortex or alignment of the implant in relative varus was detected intra-operatively or on the subsequent radiographs. Three superficial wound infections occurred, but were treated by antibiotics and all resolved. There were no other complications.
The mean navigation time for the entire series was 18.9 minutes (11 to 50 ). There was a significant difference in the navigation time for the first 20 cases (mean 26.8 minutes, 13 to 50) when compared with the remainder of the series (mean 16.9 minutes, 11 to $29, \mathrm{p}<0.002$, analysis of variance (ANOVA)). There was a trend toward decreasing navigation time for the first three consecutive series of 20 cases (Fig. 6). The mean time for navigation for the last 20 cases in the series was 15.8 minutes (12 to 27 ). There were no significant differences between the planned stem-shaft angle and that after operation when compared between the first 20 cases and the rest of the series ( $p>0.562$, ANOVA; Fig. 6 ).

\section{Discussion}

Hip resurfacing has gained popularity as a viable alternative to THR, but it is essential to adhere to strict guidelines in the selection of patients and surgical technique to ensure its continued success. Fracture of the neck of the femur remains the most common cause for revision ${ }^{35}$ with notching of the neck and varus alignment of the implant identified as major mechanical precursors of fracture. ${ }^{1,8,17}$ Computer navigation may reduce the incidence of malpreparation of the head and improve the accuracy of placement of the femoral component while reducing the learning curve and the incidence of outliers. ${ }^{18,22-28,36}$ Using cadavers, Davis et $\mathrm{al}^{18}$ showed that imageless navigation provided a range of error of almost half that of conventional instrumentation $\left(8^{\circ} \mathrm{vs} 15^{\circ}\right)$ in the placement of the initial guidewire. They also showed that the use of a conventional jig placed the guidewires in a varus position relative to the planned stem-shaft angle in all specimens. In a similar study in cadavers, Hodgson et $\mathrm{al}^{23}$ demonstrated that navigation provided markedly better repeatability in coronal alignment, compared with conventional instrumentation, 
independent of the experience of the surgeon. Using a series of 20 inexperienced medical students, Cobb et $\mathrm{al}^{36}$ assessed the accuracy of placement of the guidewire in synthetic femora by conventional jigs, CT-based pre-operative planning and CT-based intra-operative navigation. The range of error for the placement of the guidewire using conventional instrumentation was $23^{\circ}$, with a similar result achieved for CT-based pre-operative planning, while CT-based navigation yielded error of only $7^{\circ}$. Not only was navigation more accurate, but the participants using navigation were able to achieve an expert level of accuracy quickly, indicating a reduction in the length of the learning curve associated with hip resurfacing.

Several recent studies have demonstrated promising results for using imageless computer navigation over conventional instrumentation in accurate placement of the femoral component. ${ }^{21,25,27,28}$ Resubal and Morgan ${ }^{25}$ showed, in 45 navigated hip resurfacings that the range of error from the planned stem-shaft angle was within $\pm 5^{\circ}$ in all cases. By contrast, with conventional jigs only 100 of 131 hips $(76 \%)$ had a stem-shaft angle which fell within this range. They also noted that notching was avoided in the navigated group but occurred three times in those without navigation. Similarly, Ganapathi et $\mathrm{al}^{21}$ showed that in 51 consecutive navigated hip resurfacings, the range of error from the planned stem-shaft angle did not exceed $\pm 5^{\circ}$, while of 88 consecutive procedures using a standard jig, only 55 fell within this range. Again, notching was avoided in the navigated group whereas it occurred four times in the conventional series.

In our study using navigation, $86 \%$ of cases fell within $\pm 5^{\circ}$ of the planned stem-shaft angle, with an overall range of $13^{\circ}\left(5^{\circ}\right.$ of varus to $8^{\circ}$ of valgus), with no notching of the neck, while $14 \%$ erred in more than $5^{\circ}$ of relative valgus. The large number of cases may provide a realistic indication of the variability which may be encountered clinically. The pre-operative planning for estimation of the axes of the diaphysis and the neck follows that described in the literature. ${ }^{29-31,37,38}$ We attempted to harmonise the estimation of the anatomical axes to the computer algorithm which estimated these axes based on selection of anatomical landmarks and surface registration. It is important that the preoperative plan is as accurate as possible in order to match the data obtained intra-operatively and thus yield accurate insertion of the initial guidewire.

Stem-shaft angles which exceeded those planned before operation by more than $5^{\circ}$ in our study may be the result of malposition of the patient during radiography combined with variation in registration of the anatomical landmarks. During registration, the points at the two epicondyles and the piriformis fossa are used to delineate the diaphyseal axis. Deviation of the registered axis from that drawn on the pre-operative radiograph probably contributes a degree of error when drilling the guidewire using the planned stem-shaft angle. If the position of the femur is identical between the pre- and post-operative radiographs, this error may contribute to the spread of stem-shaft angles observed post-operatively. If there is deviation in the position between the pre- and post-operative radiographs, this can compound the error. As a result of the degenerative condition of the hip, the patient may not be able to carry out internal rotation adequately in order to reduce the effect of external rotation and anteversion of the femoral neck. However, several months after operation, better internal rotation allows neutralisation of the anteversion of the neck giving a better representation of the true anatomy. If this is the case, the pre-operatively assessed neck-shaft and the planned stem-shaft angles will be an overestimate and the post-operative stem-shaft angle will then be greater than expected. In a comparable navigated hip resurfacing series, Romanowski and Swank ${ }^{26}$ noted a similar phenomenon of elevated stem-shaft angles as measured by post-operative radiographs when compared with intra-operative verification of the guidewire. They also noted a possible discrepancy between the intra-operative registration of anatomical landmarks and those assessed by an AP radiograph.

However, our clinical series has shown the accuracy of navigation in achieving the desired position of the femoral component within an acceptable range of error, while avoiding preparatory errors typical of the procedure.

There was a significant improvement in the navigation time in the first 20 cases compared with the remainder of the series, with a trend towards a decrease in the time for the first three consecutive intervals of 20 cases. Improvement appeared to level off after approximately 60 navigated procedures.

Of greater significance in evaluation of the learning curve was the absence of a significant improvement in the accuracy of the coronal alignment of the femoral component. There were no significant differences between the planned and final stem-shaft angles throughout our series. This suggests that there may be a learning curve for the technique of registration but not for the overall accuracy of placement of the implant. Cobb et $\mathrm{al}^{36}$ also showed that a high level of accuracy in placement of the initial guidewire could be achieved even for novice users on their first attempt using navigation. A number of surgical fellows and trainees participated in the registration process throughout the duration of our series.

Various authors have made suggestions as to the optimal alignment of the femoral component. ${ }^{1,10-12,14}$ We plan to achieve between $5^{\circ}$ and $10^{\circ}$ of relative valgus to the native neck-shaft angle. This figure is supported by both clinical and biomechanical analyses. ${ }^{32-34}$ Fractures of the neck occur most often within the first few months after surgery. ${ }^{1,8,11,15}$ We did not experience a fracture of the neck and this provides support for the merits of navigation in facilitating optimal preparation of the head and thus reducing the risk of post-operative fracture.

There were several limitations in our study. The preoperative planning and post-operative radiological measurements were performed by a single observer which may 
introduce an error in measurement. However, post-operative measurement of the stem-shaft angle has been shown to be very repeatable with a degree of error of measurement of $1.5^{\circ} .{ }^{39} \mathrm{In}$ addition, three post-operative measurements were conducted on the pre-discharge films. Malpositioning of the patient is more likely to occur during this early radiography, and while a protocol for positioning was used in obtaining all the post-operative radiographs, we accept the limitation of measuring angulation of the component from these AP views. We only considered alignment of the implant in the coronal plane, thus limiting the conclusions which could be drawn. The use of digital AP unilateral radiography of the hip has been shown to be a reliable method for the assessment of the stem-shaft angle after operation in hip resurfacing ${ }^{39}$ but has not been demonstrated for lateral radiography in the assessment of the version of the femoral component. While this has been assessed by lateral radiographs in the clinical setting, ${ }^{25}$ there have been no studies examining the impact of femoral rotation and malposition of the patient on lateral radiographs assessing alignment of the implant after hip resurfacing. Further work is required to establish a repeatable method of assessment of version by lateral radiographs in order to support the benefit of imageless computer navigation better in the placement of the femoral component in the transverse plane of the femoral neck.

We conclude that computer navigation for placement of the initial guidewire in hip resurfacing has been shown to be more accurate than the use of conventional mechanical jigs. ${ }^{18,21,23-25,28}$ Imageless computer navigation shows promise in optimising the preparation of the femoral head and reducing the introduction of mechanical preparatory factors which predispose to fracture of the neck.

No benefits in any form have been received or will be received from a commer cial party related directly or indirectly to the subject of this article.

\section{References}

1. Amstutz HC, Beaulé PE, Dorey FJ, et al. Metal-on-metal hybrid surface arthro plasty: two to six-year follow-up study. J Bone Joint Surg [Am] 2004;86-A:28-39.

2. Back DL, Dalziel R, Young D, Shimmin A. Early results of primary birmingham hip resurfacings: an independent prospective study of the first 230 hips. J Bone Join Surg [Br] 2005;87-B:324-9.

3. Daniel J, Pynsent PB, McMinn DJ. Metal-on-metal resurfacing of the hip in patients under the age of 55 years with osteoarthritis. J Bone Joint Surg [Br]2004;86B:177-84.

4. Hing CB, Back DL, Bailey $\mathbf{M}$, et al. The results of primary birmingham hip resurfacings at a mean of five years: an independent prospective review of the first 230 hips. J Bone Joint Surg [Br] 2007;89-B:1431-8.

5. Mont MA, Seyler TM, Ulrich SD, et al. Effect of changing indications and techniques on total hip resurfacing. Clin Orthop 2007;465:63-70.

6. Steffen RT, Pandit HP, Palan J, et al. The five-year results of the birmingham hip resurfacing arthroplasty: an independent series. J Bone Joint Surg [Br] 2008;90 B:436-41.

7. Treacy RB, McBryde CW, Pynsent PB. Birmingham hip resurfacing arthroplasty: a minimum follow-up of five years. J Bone Joint Surg [Br] 2005;87-B:167-70.

8. Amstutz HC, Campbell PA, Le Duff MJ. Fracture of the neck of the femur after surface arthroplasty of the hip. J Bone Joint Surg [Am]2004;86-A:1874-7.

9. Beaulé PE, Dorey FJ, LeDuff M, Gruen T, Amstutz HC. Risk factors affecting outcome of metal-on-metal surface arthroplasty of the hip. Clin Orthop 2004;418:87-93.

10. Beaulé PE, Amstutz HC. Orientation of the femoral component in surface arthroplasty of the hip. J Bone Joint Surg [Am] 2005;87-A:1162.
11. De Smet KA. Belgium experience with metal-on-metal surface arthroplasty. Orthop Clin North Am 2005;36:203-13.

12. Freeman MA. Total surface replacement hip arthroplasty. Clin Orthop 1978;134:2-4.

13. Marker DR, Seyler TM, Jinnah RH, et al. Femoral neck fractures after metal-onmetal total hip resurfacing: a prospective cohort study. J Arthroplasty 2007;22:66-71.

14. McMinn D, Daniel J. History and modern concepts in surface replacement. Proc Inst Mech Eng [H]2006;220:239-51.

15. Shimmin AJ, Back D. Femoral neck fractures following birmingham hip resurfacing: a national review of 50 cases. J Bone Joint Surg [Br] 2005;87-B:463-4.

16. Shimmin AJ, Bare J, Back DL. Complications associated with hip resurfacing arthroplasty. Orthop Clin North Am 2005;36:187-93.

17. Siebel T, Maubach S, Morlock MM. Lessons learned from early clinical experience and results of 300 ASR hip resurfacing implantations. Proc Inst Mech Eng $[\mathrm{H}]$ 2006;220:345-53

18. Davis ET, Gallie P, Macgroarty K, Waddell JP, Schemitsch E. The accuracy of image-free computer navigation in the placement of the femoral component of the birmingham hip resurfacing: a cadaver study. J Bone Joint Surg [Br] 2007;89-B:55760

19. Barrett AR, Davies BL, Gomes MP, et al. Preoperative planning and intraoperative guidance for accurate computer-assisted minimally invasive hip resurfacing surgery. Proc Inst Mech Eng [H] 2006;220:759-73.

20. Barrett AR, Davies BL, Gomes MP, et al. Computer-assisted hip resurfacing surgery using the acrobot navigation system. Proc Inst Mech Eng [H] 2007;221:773-85.

21. Ganapathi M, Vendittoli PA, Lavigne M, Gunther KP. Femoral component positioning in hip resurfacing with and without navigation: Clin Orthop 2008;Epub.

22. Hess T, Gampe T, Kottgen C, Szawlowski B. Intraoperative navigation for hip resurfacing: methods and first results. Orthopade 2004;33:1183-93 (in German).

23. Hodgson A, Helmy N, Masri BA, et al. Comparative repeatability of guide-pin axis positioning in computer-assisted and manual femoral head resurfacing arthroplasty. Proc Inst Mech Engh [H] 2007;221:713-24.

24. Hodgson AJ, Inkpen KB, Shekhman M, et al. Computer-assisted femoral head resurfacing. Comput Aided Surg 2005;10:337-43.

25. Resubal JR, Morgan DA. Computer-assisted vs conventional mechanical jig technique in hip resurfacing arthroplasty. J Arthroplasty 2008; Epub.

26. Romanowski JR, Swank ML. Imageless navigation in hip resurfacing: avoiding component malposition during the surgeon learning curve. J Bone Joint Surg [Am] 2008:90-A:65-70.

27. Schnurr C, Michael JW, Eysel P, Konig DP. Imageless navigation of hip resurfacing arthroplasty increases the implant accuracy. Int Orthop 2007; Epub.

28. Seyler TM, Lai LP, Sprinkle DI, Ward WG, Jinnah RH. Does computer-assisted surgery improve accuracy and decrease the learning curve in hip resurfacing?: a radiographic analysis. J Bone Joint Surg [Am] 2008;90-A(Suppl 3):71-80.

29. Bobroff ED, Chambers HG, Sartoris DJ, Wyatt MP, Sutherland DH. Femoral anteversion and neck-shaft angle in children with cerebral palsy. Clin Orthop 1999;364:194-204

30. Burr DB, Cook LT, Martin NL, Asher M. Measurement accuracy of proximal femoral geometry using biplanar radiography. J Pediatr Orthop 1981;1:171-9.

31. Kay RM, Jaki KA, Skaggs DL. The effect of femoral rotation on the projected femoral neck-shaft angle. J Pediatr Orthop 2000;20:736-9.

32. Anglin C, Masri BA, Tonetti J, Hodgson AJ, Greidanus NV. Hip resurfacing femoral neck fracture influenced by valgus placement. Clin Orthop 2007:465:71-9.

33. Davis ET, Olsen M, Zdero R, Waddell JP, Schemitsch EH. Femoral component alignment and the risk of femoral neck fracture following hip resurfacing. American Academy of Orthopaedic Surgeons 2007 Annual Meeting Podium Presentations. Podium No 127. http://www3.aaos.org/education/current/aumt2007/podium/ podium.cfm?Prevent=127 (date last accessed 14 November 2008).

34. Richards CJ, Giannitsios D, Huk OL, et al. Risk of periprosthetic femoral neck fracture after hip resurfacing arthroplasty: valgus compared with anatomic alignment: a biomechanical and clinical analysis. J Bone Joint Surg [Am] 2008;90-A:96-101.

35. No authors listed. Australian Orthopaedic Association National Joint Replacement Registry 2007. http://www.aoa.org.av/docs/njavep07.pdf (date last accessed 14 November 2008).

36. Cobb JP, Kannan V, Brust K, Thevendran G. Navigation reduces the learning curve in resurfacing total hip arthroplasty. Clin Orthop 2007;463:90-7.

37. Haspl M, Bilic R. Assessment of femoral neck-shaft and antetorsion angles. Int Orthop 1996;20:363-6.

38. Yoshioka Y, Siu D, Cooke TD. The anatomy and functional axes of the femur. $J$ Bone Joint Surg [Am] 1987;69-A:873-80.

39. OIsen M, Davis ET, Gallie PA, Waddell JP, Schemitsch EH. The reliability of radiographic assessment of femoral neck-shaft and implant angulation in hip resurfacing arthroplasty. J Arthroplasty 2008; Epub. 